\section{PSICOLOGIA IBEROAMERICANA}

\section{Psicología lberoamericana}

ISSN: 1405-0943

psicología.iberoamericana@uia.mx

Universidad Iberoamericana, Ciudad de

México

México

Valdés Cuervo, Ángel Alberto; Martínez Cebreros, Marisol; Vales García, Javier

Percepciones de Docentes con respecto a la Disciplina en la Escuela

Psicología Iberoamericana, vol. 18, núm. 1, enero-junio, 2010, pp. 30-37

Universidad Iberoamericana, Ciudad de México

Distrito Federal, México

Disponible en: http://www.redalyc.org/articulo.oa?id=133915936004

Cómo citar el artículo

- Número completo

- Más información del artículo

- Página de la revista en redalyc.org

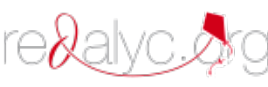

Sistema de Información Científica

Red de Revistas Científicas de América Latina, el Caribe, España y Portugal

Proyecto académico sin fines de lucro, desarrollado bajo la iniciativa de acceso abierto 


\title{
Percepciones de Docentes con respecto a la Disciplina en la Escuela
}

\author{
Teachers' Perceptions regarding School Discipline
}

\author{
Ángel Alberto Valdés Cuervo \\ Marisol Martínez Cebreros \\ Javier Vales García * \\ Instituto TeCNOLÓGico de SONORA, México
}

\section{RESUMEN}

Se realizó un estudio fenomenológico de corte cualitativo con el propósito de describir las percepciones de docentes de secundaria acerca de las causas de los problemas de disciplina de los alumnos, las estrategias que utilizan para su manejo y sus necesidades de capacitación al respecto. Para tal fin se utilizó un muestreo de casos típicos, seleccionándose a 22 docentes para participar en el estudio. La recolección de información se llevó a cabo con entrevistas a profundidad y un grupo de enfoque. Los resultados evidenciaron que los docentes poseen una visión amplia aunque poco profunda de las causas de los problemas de disciplina de los estudiantes, que incluyen su gestión entre los factores que afectan la disciplina en el aula y que utilizan estrategias convencionales tales como el castigo y la negociación para el manejo de ella.

Descriptores: Percepción, disciplina, docentes, atribuciones, intervención.

\section{ABSTRACT}

A phenomenological study was conducted with a qualitative approach in order to describe the perceptions of middle school's teachers about the causes of the student's discipline problems, and the strategies used for management and training needs regarding this issue. We used a sampling of typical cases and selected 22 teachers to participate in the study. The information was gathered through in-depth interviews and a focus group. The results showed that teachers have a broad but shallow view of the causes of discipline problems in students, which includes its management among the factors that affect discipline in the classroom and the use of conventional strategies such as punishment and negotiation for the management of discipline.

Keywords: Perception, discipline, teachers, attributions, intervention.

\footnotetext{
* Para correspondencia: Ángel Alberto Valdés Cuervo. Maestro en Investigación Educativa. Departamento de Educación. Instituto Tecnológico de Sonora. Calle 5 de Febrero 818 Sur, Centro, Ciudad Obregón, Sonora. Correo electrónico: avaldes@itson.mx. Tel: (644) 41009 27. • Marisol Martínez Cebreros. Estudiante de la Licenciatura en Ciencias de la Educación. Departamento de Educación. Instituto Tecnológico de Sonora. Calle 5 de Febrero 818 Sur, Centro, Ciudad Obregón, Sonora. Correo electrónico: marisolcebreros@hotmail.com Tel: (644) 41009 27. • Javier José Vales García. Doctor en Educación. Instituto Tecnológico de Sonora. Calle 5 de Febrero 818 Sur, Centro, Ciudad Obregón, Sonora. Correo electrónico: jvales@itson.mx. Tel: (644) 4100921.
} 


\section{INTRODUCCIÓN}

El aula es un espacio donde se manifiestan valores, ideas e intereses diferentes y a menudo contradictorios. Por lo tanto, es un lugar donde continuamente se generan conflictos que deben ser negociados para mantener un adecuado clima escolar.

La disciplina en la escuela no solo dificulta o favorece el aprendizaje, sino que, además, es un medio que favorece la creación de espacios de discusión, participación y generación colectiva de nuevas normas en el momento en que son necesarias (Sús, 2005). Por otra parte, contribuye a evitar fenómenos como la violencia escolar, que afectan nocivamente tanto a los que la padecen como a los que la perpetran.

A pesar del reconocimiento de la importancia de la disciplina como elemento pedagógico, los problemas de falta de ella en las escuelas son cada vez más frecuentes y resulta obvio que en muchas ocasiones existen dificultades para manejarlos, lo que provoca que se enquisten y que las dificultades conductuales crezcan cada día más (Pastor, 2002; Sala, 2001).

La disciplina, que podía ser considerada hasta hace poco tiempo un tema secundario en el orden de prioridades de las preocupaciones de los docentes, ha pasado a ocupar un lugar de primer orden entre ellas (Gotzens, Castello, Genovard \& Badía, 2003). Un número importante de profesores ubican en los problemas de comportamiento de los alumnos la principal barrera con la que se topan en el desarrollo de su ejercicio profesional (Cubero, 2004); a pesar de los esfuerzos, tanto individuales como colectivos, de los docentes, por el momento no parece que los problemas de disciplina se encuentren en un camino con garantías de solución.

Esta situación se evidencia en el estudio que realizó el Instituto Nacional de Evaluación Educativa ([INEE], 2006), acerca de la violencia en las escuelas, donde se comprobó que $11 \%$ de los alumnos reconoce haber participado en peleas; $43.6 \%$ refiere que en las escuelas les han robado; $14 \%$ haber sido lastimado físicamente por otro alumno; y $13.6 \%$ haber recibido burlas de parte de sus compañeros.

Otros estudios que ilustran la situación con respecto a la disciplina que prevalece en las escuelas mexicanas son los que realizó Velásquez (2005), quien refiere que $64 \%$ de los estudiantes de bachillerato reportaron abuso de sus compañeros a lo largo de su vida escolar, y 76\% acusa violencia verbal por parte de los docentes. Por otra parte, Castillo y Pacheco (2008), reportaron que $52.8 \%$ de los estudiantes de secundaria referían haber padecido apodos ofensivos, $48.8 \%$ insultos, $22.2 \%$ golpes y $22 \%$ exclusión social.

Los problemas de disciplina ocasionan la degradación del clima escolar, entendido éste como la calidad general de las relaciones e interacciones entre los diferentes actores de la escuela (Blaya, 2003). Por otra parte, Galloway (2003) sostiene que el clima escolar ejerce una poderosa influencia en el desarrollo intelectual, psicológico y social de los estudiantes, por lo que su deterioro afecta negativamente en ellos; Sánchez (2003) reporta que en los problemas de disciplina influyen múltiples factores, los cuales pueden ser de índole contextual (características socioeconómicas y culturales de la comunidad y la familia), de índole individual (particularidades de carácter subjetivo de los estudiantes y de sus interacciones), y relativos a la escuela (clima escolar) (Sánchez, 2003).

El docente juega un papel importante en el manejo de la disciplina, ya que es el representante de la ley y quien está investido del poder y la responsabilidad de mantener el orden en la clase. Según Gotzens (1997), dentro de las habilidades que debe poseer el docente se encuentran las relacionadas con el manejo de la disciplina, es decir, las que favorecen el desarrollo armónico de las relaciones de los miembros del grupo entre ellos y con él.

La manera en que perciben y representan los problemas de disciplina los docentes es una de las claves que explica lo que es en realidad y cómo se vive la disciplina en el aula (Castelló, 2002). Esto se debe a que toda percepción comporta una interpretación, por lo que más que tratarse de un fenómeno puramente sensorial, es necesario considerarlo como una actividad psicológica compleja, vinculada a un cuadro de referencias particular (Castelló, 2001).

Por otro lado, la manera en que interpretamos el mundo influye en la forma en que lo comprendemos, sentimos y actuamos en él (Gergen, 1996). Se justifica de esta manera estudiar las percepciones que tienen los docentes acerca de los problemas de disciplina, ya que las ideas que poseen acerca de lo que es admisible o no dentro de un aula, favorecedor o disruptivo del proceso instruccional, influye en la calidad del clima que logran promover en sus salones de clases. 
Definición y funciones de la disciplina

García (2008) sostiene que la disciplina constituye un elemento del proceso de enseñanza-aprendizaje, a través del cual los alumnos aprenden a vivir como miembros de una sociedad, por lo que se presenta como una cuestión relevante en el contexto educativo.

En el aula, la disciplina se elige como un recurso para conseguir determinados fines, como la socialización del alumnado, la autonomía, el rendimiento académico y el autocontrol. En cualquier caso, se trata de un concepto debatido en el que influyen dos formas de entenderla: por un lado, la disciplina como correctora de conductas inadecuadas y, por otro, como una estrategia para desarrollar y controlar las conductas deseadas (García, 2006).

En este mismo sentido, Gotzens, Castello, Genovard y Badía (2003), la entienden como el conjunto de procedimientos, que incluye normas o reglas, mediante las cuales se mantiene el orden en la escuela y cuyo valor no es otro que el de favorecer la adquisición de los objetivos propuestos en el proceso enseñanzaaprendizaje del alumno.

Por ello, la disciplina es útil para manejar los problemas de conductas de los alumnos que interfieren o dificultan la actividad normal del profesor en el aula. También es un instrumento eficaz para lograr que el alumno sea capaz de controlar su propia conducta, desarrollar el sentido de autocontrol, incrementar la autoestima y promover su sentimiento de seguridad personal (Pastor, 2002).

Según García (2008), la necesidad de la disciplina se fundamenta en criterios de diversa índole: a) Social, pues ninguna sociedad, por muy sencilla y rudimentaria que sea, puede funcionar sin normas. La escuela, como institución social, debe cumplir normas de respeto, convivencia y poseer control sobre el cumplimiento de las mismas; b) Pedagógica, ya que la educación tiene como función disciplinar la mente, la imaginación y la atención y para conseguirlo es fundamental y básico el orden, la gestión y el control para hacer personas disciplinadas y respetuosas; c) Psicológica, debido a que parte del supuesto de que el adolescente necesita de un orden y disciplina para ser feliz y aceptado socialmente, y lograr un sano desarrollo psicológico.

\section{El docente y la disciplina}

Como ya se mencionó, los problemas de disciplina tienen múltiples influencias, pero, de manera casi uniforme, diversos autores hacen hincapié en que la naturaleza de la gestión docente es un factor importante para establecer una adecuada disciplina (Fierro, 2005; Sús, 2005; Vázquez, Villanueva, Rico \& Ramos, 2005). A pesar de la importancia que tiene el docente en la promoción de la disciplina, varios estudios muestran que los maestros tienden a negar su propia influencia en los problemas de disciplina, pues atribuyen las causas a factores externos a la escuela, como al contexto, la familia y el propio estudiante (Palomero \& Fernández, 2001, Saavedra, Villata \& Muñoz, 2007).

Por otra parte, por lo general los docentes no cuentan con estrategias eficaces para manejar los problemas de disciplina dentro de sus salones de clase y tienden a adoptar una actitud autoritaria o permisiva, esto es, utilizan pocas veces un estilo democrático para el manejo de las situaciones que se les presentan en el desarrollo de sus tareas (Sús, 2005).

Con respecto a lo anterior, en un estudio que realizó Sús (2005) se comprobó que, por lo general, el manejo de las normas por parte de los maestros se realiza de tres formas: a) mediante la inflexibilización o rigidización de las normas; en este caso se procura el cumplimiento de la norma sin que los alumnos comprendan su verdadera función y se utiliza el castigo como principal estrategia correctora; b) A través del establecimiento de normas inconsistentes; las normas no son claras, cambian de una situación a otra y no se coordinan en torno a la tarea, lo que da origen a una total informalidad y faltas de respeto; y c) Por medio del debilitamiento del cumplimiento de la norma; en esta situación, rara vez los maestros intervienen para regular las normas de convivencia entre los estudiantes.

El análisis de los planteamientos de varios autores tales como Sús (2005), Chagas (2005), Saucedo y Furlan (2005) permite afirmar que dentro de la escuela existen dificultades para manejar los problemas de disciplina tales como: a) persistencia de un modelo autoritario en el manejo de la autoridad; b) falta de consensos e inconsistencias acerca de las normas de disciplina; c) uso del castigo y especialmente de la acreditación como el único instrumento para el manejo de la disciplina, y d) la existencia de impunidad dentro de las escuelas, 
situación que lleva a la frecuente infracción y naturalización de los problemas de disciplina.

\section{OBJETIVO DEL ESTUDIO}

Mediante este estudio se pretende contribuir a la comprensión del fenómeno de la disciplina a través de las percepciones que con respecto a ella poseen los docentes, ya que ellos juegan un papel importante en su conceptualización y manejo.

\section{Preguntas críticas de investigación}

1. ¿A qué causas atribuyen los docentes los problemas de disciplina?

2. ¿Qué estrategias utilizan los docentes para manejar a un estudiante con problemas de disciplina en el salón de clases?

3. ¿Qué tipo de capacitación los docentes consideran necesitar para manejar eficazmente a los estudiantes con problemas de disciplina?

\section{MÉTODO}

\section{Tipo de estudio}

Se realizó un estudio cualitativo de tipo fenomenológico, ya que su propósito fue describir las percepciones de los docentes de secundaria acerca de los problemas de disciplina.

\section{Participantes}

Se utilizó un muestreo por casos típicos de docentes de una secundaria pública del sur de Sonora. El criterio de caso típico condujo a que se seleccionaran a los maestros de planta de dicha escuela, que constituían $76 \%$ del total de la planta docente e imparten $80 \%$ de las horas clase. En total participaron 22 docentes, con una edad promedio de 47 años, con un máximo de 57 y un mínimo de 25 años. Poseían en promedio 20 años de servicio con un mínimo de 3 y un máximo de 31 años. De ellos, 91\% eran egresados de la escuela Normal Superior.

\section{Técnicas utilizadas}

Se aplicaron entrevistas a profundidad y se utilizó un grupo de enfoque. Las entrevistas a profundidad se utilizaron para adentrarse en las perspectivas de los docentes acerca de la temática de la disciplina, mien- tras que se recurrió al grupo de enfoque para ampliar la información que se obtuvo mediante las entrevistas y validarla.

\section{Métodos de verificación y validación}

Para validar la información, las entrevistas fueron analizadas por varios expertos y posteriormente se le presentaron los resultados de los análisis a los mismos docentes para que ellos los validaran.

\section{Aspectos éticos}

Se procuró la participación informada y voluntaria de los docentes; se tomaron medidas para garantizar la confidencialidad de la información y validarla con los propios docentes.

\section{RESULTADOS}

Para el análisis de los resultados se presentaron los ejes temáticos y las categorías de análisis generadas en cada uno de ellos.

\section{Causas a que atribuyen los docentes los problemas de disciplina}

En este eje temático se generaron cuatro categorías de análisis basadas en que los docentes atribuyen los problemas de disciplina a características del contexto social del estudiante, de su familia, del propio estudiante, y de la escuela y los docentes.

Relacionados con el contexto social. Los docentes señalan como causas de los problemas de disciplina características de la sociedad tales como la violencia social generalizada y los fenómenos particulares del contexto, como la existencia de bandas juveniles. También destacan la influencia de los medios de comunicación, lo cual se ilustra en lo que expresa uno de los maestros.

Caso 20: "El medio social donde ellos viven influye si es un lugar donde existe la propagación de pandillerismo o tribus, pues el alumno también va a tener ese comportamiento aquí en la escuela”.

Relacionadas con la familia. Según los docentes las familias de los estudiantes contribuyen a generar e incrementar sus problemas de disciplina, ya que en ellas existen problemas económicos y desintegración; ade- 
más, presentan problemas de adicciones, violencia y desatención por parte de los padres. Para la ilustrar esta característica se transcribe lo que expresaron algunos docentes en las entrevistas y en el grupo de enfoque.

Caso 9: "Hay muchas razones pero principalmente yo creo que son los problemas familiares, ya que son alumnos que vienen de hogares desintegrados".

Relacionados con el alumno. Los docentes consideran que las características de los estudiantes que favorecen la presencia de problemas de disciplina son el desinterés por la escuela, las bajas expectativas académicas, la hiperactividad y los problemas de aprendizaje. Al respecto, uno de los docentes comentó:

Caso 5: "Alumnos con problemas de hiperactividad, o alumnos que acarrean un problema de aprendizaje desde la primaria; hay alumnos que no saben leer ni escribir en tercer grado".

Relacionados con la escuela y los docentes. Para los maestros, algunas características de la escuela contribuyen a la presencia de problemas de disciplina. Entre ellas de destacan los insuficientes servicios de apoyo de orientación psicológica, el ambiente escolar inadecuado y la presencia de estudiantes con problemas de conducta en la escuela.

Reconocen también que características de ellos mismos como docentes pueden contribuir la presencia de problemas de disciplina, a saber: la poca motivación por la enseñanza, la falta de estrategias para el manejo de los problemas de disciplina en el salón de clases y las estrategias didácticas poco motivadoras para el estudiante, situación que se ilustra con los siguientes planteamientos de los profesores.

Caso 5: "Como docentes, nosotros no estamos preparados para tratar a un alumno con alguna discapacidad como alumnos con problemas de aprendizaje o hiperactividad; esto se debe a que no tenemos la formación ni la información de cómo tratar al estudiante con problemas".

\section{Estrategias que utilizan los docentes para manejar los problemas de disciplina}

En este eje temático se generaron cuatro categorías de análisis con base en que los docentes manifiestan que para manejar los problemas de disciplina utilizan es- trategias tales como: apoyos externos, castigos, negociación con los estudiantes y referidas a la organización de la clase.

Relacionadas con apoyos externos. Los docentes manifestaron que recurren a los padres de familia o a especialistas para que los apoyen para el manejo de los estudiantes con problemas de disciplina.

Caso 1: "Cuando el alumno comete una falta llamo al prefecto o lo mando a trabajo social, y en ocasiones se mandan llamar a sus papás".

Relacionadas con castigos. Se describe la utilización de castigos para controlar a los alumnos con problemas de disciplina, en especial los relacionados con las calificaciones.

Caso 14: "Muchas veces optamos por lo mas fácil, como sacar al estudiante del aula; es quitar el problema de encima, aunque sea de manera momentánea, pues sabemos que esta solución no ayuda al adolescente”.

Negociación con el estudiante. Una estrategia que los maestros aplican es la búsqueda de acuerdos y compromisos con los estudiantes para que éstos regulen su comportamiento.

Caso 4: "Como docente, si el alumno tiene problemas de disciplina platico con él; soy de las personas que cree que se puede convencer al joven".

Relacionadas con la organización de la clase. Los maestros comentan que para controlar a los estudiantes con problemas de disciplina establecen reglas y desarrollan diversas estrategias de enseñanza.

Caso 8: "Lo que yo hago es buscar impartirle la clase de diferentes maneras; a veces hacemos trabajo en equipo o individual, otras traigo material como videos".

Tipo de capacitación que los docentes consideran necesario para manejar eficazmente a los estudiantes con problemas de disciplina La capacitación que los docentes consideran necesaria se agrupó en las siguientes categorías: particularidades del desarrollo del adolescente, detección y evaluación de estudiantes con problemas de disciplina, manejo de los problemas de disciplina, y estrategias para el desarrollo de la autoestima, valores y habilidades sociales. 
Particularidades del desarrollo del adolescente. Los docentes manifiestan que necesitan apoyo para ampliar sus conocimientos acerca de las características del adolescente, los factores que afectan su aprendizaje y los problemas que enfrentan.

Caso 6: "Cursos de cómo tratar al adolescente, sobre la autoestima, sobre cómo tratar los problemas de aprendizaje y los particularidades de la edad".

Detección y evaluación de estudiante con problemas de disciplina. Los maestros solicitan apoyo para contar con competencias que les permitan identificar y comprender mejor las características de los estudiantes con problemas de disciplina.

Caso 3: "Utilizar una prueba que nos ayude a detectar a un alumno con problema a partir de los rasgos que presenta; nos ayudaría a elaborar un expediente bien establecido".

Manejo de los problemas de disciplina. Los docentes comentan que se debe estimular el desarrollo de técnicas y estrategias que les permitan el manejo eficaz de los problemas de disciplina ante el desconocimiento que manifiestan de ellas.

Caso 11: "Yo creo que hacen falta más técnicas, ya que el alumno está en cambio permanente y nosotros muchas veces no nos actualizamos; necesitamos nuevas técnicas, nuevas formas de tratar al alumno e información de psicología para ayudarlos de manera adecuada".

Estrategias para el desarrollo de la autoestima, valores y habilidades sociales. Para manejar los problemas de disciplina los maestros también consideran necesario recibir capacitación en estrategias para el fomento de la autoestima, los valores y habilidades sociales en sus estudiantes.

Caso 17: "Cursos sobre valores, comportamientos de los adolescentes, ya que son muchos alumnos y necesitamos ayuda más especializada y estar al pendiente".

\section{DISCUSIÓN}

Se comprobó que los docentes mostraron una visión amplia de las causas de los problemas de disciplina, pues consideraron que sobre ellos influyen aspectos sociales, familiares, de los mismos estudiantes, de la escuela y de su propia práctica. El hecho de que hayan resaltado que ciertas características de su práctica pueden afectar la disciplina en el salón de clases resulta especialmente llamativo, ya que es contrario a los hallazgos de estudios que realizaron otros autores, tales como Fierro (2005), Palomero y Hernández (2001), Sús (2005), quienes refieren que los maestros tienden a atribuir los problemas de disciplina a factores externos a la escuela y a su gestión al frente de los estudiantes.

Una posible explicación de esta diferencia es que el aumento del interés en el fenómeno de la disciplina y la socialización de la información ha incrementado la comprensión de la problemática por parte de los docentes.

Sin embargo, se observó que el nivel de profundidad del análisis de los maestros de las causas es pobre, ya que sus respuestas tienden a ser estereotipadas y de sentido común, y no muestran un conocimiento profundo acerca de la forma en que se produce la influencia de la familia, el propio alumno y su propia práctica docente en los problemas de disciplina.

Cuando abordan la influencia de la familia no se refieren en ningún momento a conceptos que describen el funcionamiento familiar como son los límites, autoridad, ciclo vital y creencias familiares, conceptos que desde el punto de vista científico ayudan a explicar el funcionamiento familiar según autores como Minuchin (1974), Hoffman (1998) y Valdés, Esquivel y Artiles (2007).

Por otra parte, los docentes atribuyen a los alumnos características que no siempre están presentes en los estudiantes con problemas de disciplina (problemas de aprendizaje, falta de motivación hacia el estudio, entre otros), pero no señalan características dinámicas o de personalidad con mayor poder explicativo tales como la autoestima, las habilidades sociales, los valores, etc.

Además, cuando señalaron las características docentes que consideran como potenciales causantes de los problemas de disciplina (falta de motivación docente y estrategias didácticas poco motivadoras, importantes, por cierto) dejaron de lado estrategias concretas referidas al manejo de las normas y la autoridad en el salón de clases.

Las estrategias mencionadas por los docentes para manejar la disciplina en sus aulas hacen referencia a ayudas externas a la escuela provenientes de la familia y de profesionales de la salud mental, que indiscutible- 
mente resultan apropiadas para atacar el problema. Sin embargo, en el salón de clases se aprecia un predominio de las estrategias de tipo convencional, tales como el castigo y la negociación, ya que no incluyen entre sus posibles abordajes métodos basados en un enfoque de convivencia, que implica la participación de los estudiantes en el establecimiento de las reglas y la necesidad de que comprendan el valor de ellas.

En cuanto a las necesidades de capacitación, se observó que éstas abarcan tanto aspectos generales de la adolescencia y estrategias para la promoción de cualidades y habilidades, como el entrenamiento en elementos de diagnóstico e intervención con estudiantes indisciplinados. Estas demandas denotan la conciencia que tienen los docentes de sus limitaciones para el manejo eficaz de los problemas de disciplina, ya que sus necesidades de capacitación abarcan desde aspectos generales de formación psicológica hasta intervenciones específicas.

\section{CONCLUSIONES}

El análisis de los resultados permitió llegar a las siguientes conclusiones:

\section{REFERENCIAS}

Blaya, C. (2003). Violence á I'ecóle et socialisation professionalle: les leçons du comparatisme. Ponencia presentada en Second International Conference on Violence in School: Research, Best Practices and Teacher Training. Québec: Université Laval.

Castelló, A. (2001). Inteligencias. Una integración multidisciplinar. Barcelona: Masson.

Chagas, D. (2005). Los maestros frente a la violencia entre los alumnos. Revista Electrónica de Investigación Educativa, 10 (1). Recuperado el 21 de Marzo de 2009, de http://redie.uabc.mx/vol0no27/contenido.htm

Cubero, C. (2004). La disciplina en el aula. Costa Rica: IIMEC.

Fierro, M. (2005). El problema de la indisciplina desde la perspectiva de la gestión directiva en escuelas públicas de nivel básico. RMIE, 10 (27), 1133-1148. a) En las percepciones acerca de las causas de los problemas de disciplina, los docentes consideran la acción de varios sistemas, lo cual les da una perspectiva amplia a sus opiniones al respecto.

b) Es de importancia el hecho de que los docentes consideren que su práctica contribuye a la existencia de problemas de disciplina, ya que a partir de esta perspectiva se abre una oportunidad de trabajo con ellos en ese sentido.

c) Se evidenció que en las opiniones de los docentes acerca de las causas de los problemas de disciplina predominan estereotipos culturales que denotan pocas referencias a conceptos científicos.

d) Se comprobó que para controlar la disciplina continúan utilizándose métodos convencionales tales como el castigo y la negociación y una evidente ausencia de estrategias basadas en la convivencia que implica la participación de los propios alumnos en el establecimiento de las normas y la comprensión de éstas.

e) Que los docentes poseen conciencia de la necesidad que tienen de ser capacitados para el manejo de la disciplina en las escuelas.

Galloway, D. (2003). Should we try to reduce bullying. En Memoire du deuxieme conference mundiale sur la Violence á I'ecole, Recherche, practique exempalires et formation des maîtres. Québec.

García, C. A. (2006). La disciplina escolar. España: Universidad de Murcia.

García, C. (2008). Disciplina escolar, una guía docente. España: Universidad de Murcia.

Gergen, K. (1996). Realidades y relaciones. España: Paidós. Gotzens, C. (1997). La disciplina escolar. Barcelona: Horsori.

Gotzens, C.; Castello, A.; Genovard, C. \& Badía, M. (2003). Percepciones de profesores y alumnos de E.S.O. sobre la disciplina en el aula. Psicothema, 15 (3). Recuperado el 15 de Marzo de 2009, de http://www.psicothema. com/ 
Hoffman, L. (1998). Fundamentos de terapia familiar. Un marco conceptual para el cambio de sistemas. México: Fondo de Cultura Económica.

Instituto Nacional de Evaluación Educativa. (2006). Informe sobre La Calidad de la Educación Básica en México. Violencia y disciplina en las escuelas primarias y secundarias. México: INEE.

Minuchin, S. (1974). Familias y terapia familiar. México: Gedisa.

Palomero, J. \& Hernández, M. (2001). La violencia escolar. Un punto de vista global. Revista Interuniversitaria de Formación del Profesorado, 41, 19-38.

Pastor, B. (2002). ¿Qué pasa en las aulas? Barcelona: Editorial Planeta.

Saavedra, E., Villata, M. y Muñoz, M. (2007). Violencia escolar: la mirada de los docentes. Límite, 2 (15), 39-60.

Sala, T. (2001). Pequeña crónica de un profesor de Secundaria. Barcelona: Ediciones.
Sánchez, P. (2003). Aprendizaje y Desarrollo. México: Consejo Mexicano de Investigación Educativa.

Saucedo, C. y Furlan, A. (2005). Copetes 'EMO' y celulares en la escuela secundaria: la disciplina escolar puesta a prueba. Ponencia presentada en el X Congreso Nacional de Investigación Educativa. Veracruz: COMIE.

Sús, M. (2005). Convivencia o disciplina ¿Qué está pasando en la escuela? RMIE, 10 (27), 983-1004.

Valdés, A., Esquivel, L. \& Artiles, K. (2007). Familia y desarrollo. Estrategias de intervención en terapia familiar. México: Manual Moderno.

Velázquez, C. (2005). Experiencias estudiantiles con la violencia en la escuela. RMIE, 10 (26), 739-764.

Vázquez, R., Villanueva, A., Rico, A. y Ramos, M. (2005). La comunidad de la preparatoria 2 de la Universidad de Guadalajara. RMIE, 10 (27), 1047-1070. 